\title{
Relative Fat Mass Index can be solution for obesity paradox in coronary artery disease severity prediction calculated by SYNTAX Score
}

\author{
Süleyman Çağan Efe $\odot$, ,'Ali Karagoz, ${ }^{1}$ Cem Dogan, ${ }^{1}$ Zubeyde Bayram, \\ Sedat Kalkan, ${ }^{1}$ Mehmet Sait Altıntas, ${ }^{2}$ Yasin Yuksel, ${ }^{2}$ Turgut Karabag, ${ }^{2}$ Burak Ayca, ${ }^{2}$ \\ Nihal Ozdemir ${ }^{1}$
}

${ }^{1}$ Cardiology, Kartal Kosuyolu Training and Research Hospital, Istanbul, Turkey

${ }^{2}$ Cardiology, Istanbul Teaching and Research Hospital, Istanbul, Turkey

\section{Correspondence to} Süleyman Çağan Efe, Cardiology, Kartal Kosuyolu Training and Research Hospital, Istanbul 34865, Turkey; scaganefe@gmail.com

Received 24 August 2020 Revised 20 October 2020 Accepted 29 October 2020 Published Online First 11 December 2020
Check for updates

(C) Author(s) (or their employer(s)) 2021. No commercial re-use. See rights and permissions. Published by BMJ.

To cite: Efe SÇ, Karagoz A,

Dogan C, et al.

Postgrad Med J

2021:97:434-441

\section{ABSTRACT}

Background The relation between body mass index (BMI) and coronary artery disease (CAD) extension remains controversial. A new score was developed to estimate body fat percentage (BFP) known as Relative Fat Mass (RFM) Index. This study aimed to evaluate the value of RFM Index in predicting the severity of the $C A D$, compared with other anthropometric measurements.

Methods A total of 325 patients with chronic CAD were investigated. RFM, BFP, BMI and other anthropometric characteristics of patients were measured before angiography. CAD severity was determined by SYNergy between percutaneous coronary intervention with TAXus and cardiac surgery trial (SYNTAX) Score. The association between SYNTAX Score and variables was evaluated using linear regression models. In order to compare the model performance, R-squared $\left(R^{2}\right)$, Akaike's information criterion, Bayesian information criterion and root mean square error were used.

Results Univariate linear regression outcome variable, SYNTAX was used to determine whether there was any relationship between variables. Independent variables were included in the multivariable linear logistic regression models. The analysis showed that in model 1, RFM ( $\beta$ coefficient: 2.31 (0.90 to 3.71), $p=0.001)$ ), diabetes mellitus ( $\beta$ coefficient: 3.72 (1.67 to 3.76), $p=0.004)$ ), haemoglobin ( $\beta$ coefficient: $-2.12(-3.70$ to -0.53$)$, $p=0.03$ ) and age ( $\beta$ coefficient: 1.83 (0.29 to 3.37), $p=0.02)$ ) were statistically significant. The adjusted $R^{2}$ values in model 1 were higher than model 2 (BFP) and model 3 (BMI) $(0.155,0.137$ and 0.130 , respectively), and $\chi^{2}$ values of RFM were higher than BFP and BMI (10.5, 3.4 and 1.0, respectively).

Conclusion RFM Index is a more reliable and compatible marker of obesity in showing the severity of CAD compared to BMI.

\section{INTRODUCTION}

In recent years, dramatic increase in the prevalence of obesity has become a major health problem and reached alarming dimensions. ${ }^{1}$ It is known that being overweight and obese are associated with an increased risk of cardiovascular disease, that can cause mortality in the general population. ${ }^{23}$ Although different approaches have attempted to define obesity to date, there is no consensus about its exact definition yet. In one definition, obesity is introduced as an increase in the body fat percentage (BFP). ${ }^{4}$ Body mass index (BMI) has been used frequently to determine definitions for obesity limits. However, it is thought that it is not a sufficient formula to determine the body fat described in the definition of obesity. This situation is manifested by its inability to distinguish people with different fat ratios even though they have the same BMI values. ${ }^{6}$

Evidence from some studies in patients with obesity with coronary artery disease (CAD) appears to be contradictory. As a result of these studies, the concept of 'obesity paradox' was proposed due to the protective effect of obesity in spite of the negative consequences. ${ }^{7-10}$ Moreover, the same results as obesity paradox were confirmed for the severity of CAD in some studies. ${ }^{11-13}$ Meanwhile, some studies showed that patients with increased BMI had a greater prevalence, extent and severity of CAD. ${ }^{14}{ }^{15}$ These controversial results lead the researchers of current study to evaluate the utility of BMI in the diagnosis of obesity and its relevance to cardiovascular events.

Classification of obesity according to the BMI has been questioned in some studies. For example, some authors believe that BMI is a parameter that should be used in the follow-up of patients, and not in the diagnosis of obesity. ${ }^{16}$ In addition, some other parameters stated by international consensus are more valuable in recommending and monitoring weight loss like waist circumference (WC). ${ }^{17}$ Confusion caused by irrelevance between BMI and obesity led the researchers to seek for new equations. As a result of the studies, a new proposal was made to estimate the whole body fat (WBF) and to define obesity, which is known as relative fat mass (RFM). RFM Index is calculated using height, WC and gender regardless of weight. In some studies, it has been shown that the percentage of WBF is better predicted by RFM than BMI. ${ }^{18}$

The prevalence of $\mathrm{CAD}$ is associated with the mortality and morbidity of patients. Hence, different scoring systems were improved to determine the prevalence and severity of CAD. SYNergy between percutaneous coronary intervention with TAXus and cardiac surgery trial (SYNTAX) Score has shown prognostic value for short-term and long-term adverse cardiovascular events. ${ }^{19} 20$ 
468 Patients searched

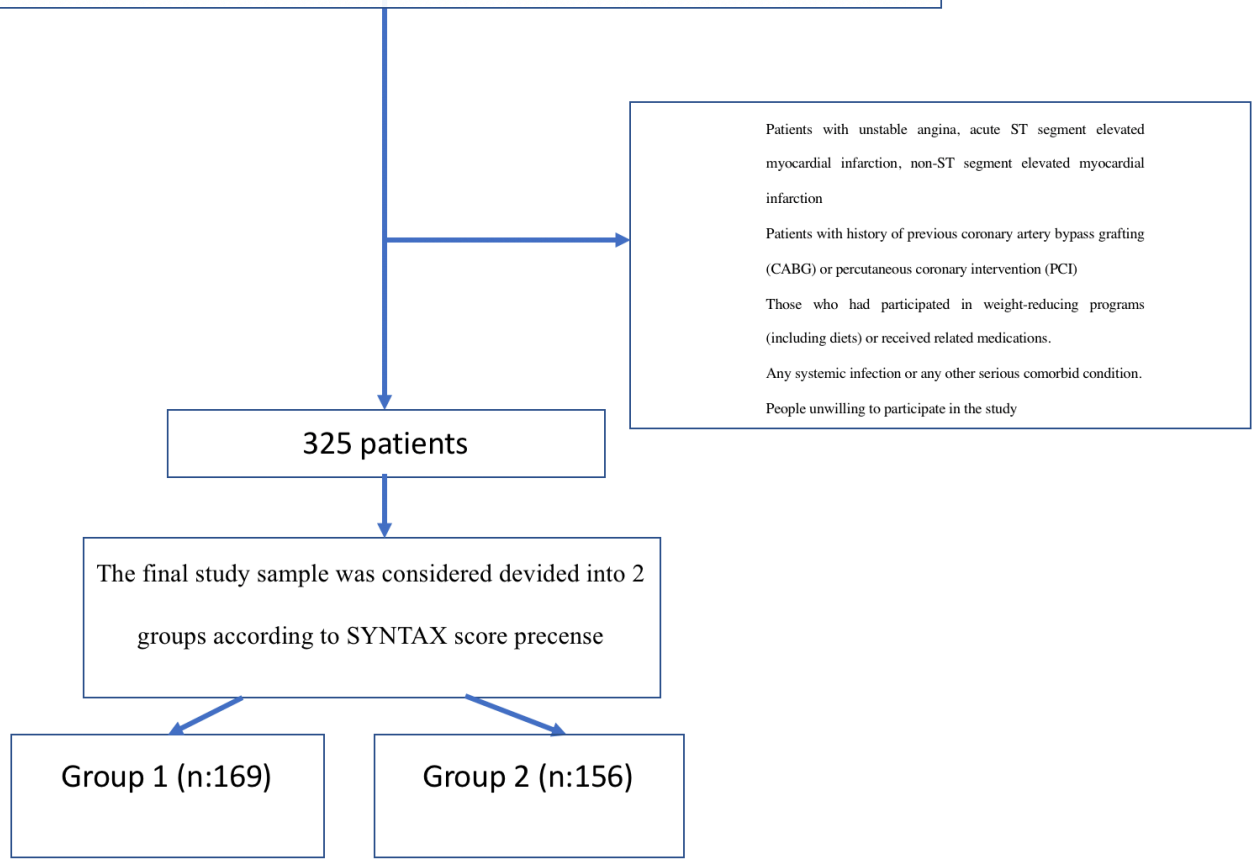

Figure 1 Consolidated Standards of Reporting Trials flow diagram of study. SYNTAX, SYNergy between percutaneous coronary intervention with TAXus and cardiac surgery trial.

Accordingly, this study was conducted to evaluate the value of RFM Index in predicting the severity of the CAD, compared with other anthropometric measurements.

\section{METHODS}

This cross-sectional study was carried out with the participation of 325 patients whose chronic CAD was determined by stress electrocardiography or myocardial perfusion scintigraphy. The samples had been admitted to an outpatient cardiology clinic from June 2018 to October 2020.

\section{Inclusion criteria}

There were two inclusion criteria: subjects aged between 18 and 80 years and patients with chronic stable angina and undergoing first time coronary angiography (CAG) for CAD detection.

\section{Exclusion criteria}

There were five exclusion criteria as follows:

1. Patients with unstable angina, acute ST segment-elevated myocardial infarction, non-ST segment-elevated myocardial infarction.

2. Patients with history of previous coronary artery bypass grafting or percutaneous coronary intervention (PCI).

3. Those who had participated in weight-reducing programmes (including diets) or received related medications.

4. Any systemic infection or any other serious comorbid condition.

5. People unwilling to participate in the study.

The inclusion and exclusion criteria have been summarised in the Consolidated Standards of Reporting Trials flow diagram (figure 1).

\section{Measurements}

The body height was taken in the standing position close to wall without shoes; weight was measured similarly without heavy dresses before CAG. WC was measured at the mid-point between the distal border of the ribs and the top of the iliac crest with subjects standing at the end of a normal expiration. BMI was calculated by dividing body weight in kilograms by the square of body length in metres $\left(\mathrm{kg} / \mathrm{m}^{2}\right)$. Waist height ratio was calculated by dividing WC by height $(\mathrm{cm}) .^{21}$

By using the US Navy method, the BFP formula was calculated as follows: ${ }^{21}$

BFP (women) $=495 /\left(1.29579-0.35004 * \log _{10}\right.$ (Waist

+ Hip Neck) $+0.22100 * \log _{10}$ (Height) $)-450$;

BFP $($ men $)=495 /\left(1.29579-0.35004 * \log _{10}\right.$ (Waist Neck $)+0.22100 * \log _{10}($ Height $\left.)\right)-450$; and

RFM Index was defined according to the following equation: $64-(20 \times$ height/waist $)+(12 \times$ sex $),($ sex $=0$ for men and 1 for women). ${ }^{18}$

Obesity was considered when BMI was equal or higher than $30 \mathrm{~kg} / \mathrm{m}^{2}$, RFM higher than 33.9 in women or 22.8 in men. ${ }^{21}$

In this study, we used SYNTAX 1 Score because it is based on anatomical stenosis irrespective of other parameters. Parameters which take part in SYNTAX Score 2 (diabetes mellitus (DM), creatinine (CR)) could affect our results. Since using similar parameters like DM or CR as independent predictor in regression model affects the reliability of results, we preferred to use SYNTAX Score 1. The SYNTAX I Score (www.SYNTAXscore. com) was determined according to the coronary artery stenosis and the anatomic complexity of the coronary lesions in patients with multivessel disease. SYNTAX Score showed a significant correlation with the morbidity and mortality outcomes of patients after PCI and was proposed as a tool to determine the option of revascularisation in patients with CAD. As SYNTAX 
Scores increase, the prevalence of coronary disease refers to more complex and difficult interventional procedures, thus representing potentially worse prognosis. ${ }^{19}$

The SYNTAX Score was determined by two independent cardiologists (SCE, BA) with 5 years of interventional experience. Also, Cronbach's $\alpha$ for interobserver and intraobserver reliability analysis was 0.964 and 0.982 , respectively. After independent evaluations, the possible differences in the scores were re-evaluated and unanimously resolved.

\section{STATISTICAL ANALYSIS}

All statistical analyses were performed using 'rms', 'Hmisc' and 'ggplot2' packages with R V.4.0 (R Project, Vienna, Austria). Continuous variables were expressed as mean $\pm \mathrm{SD}$; and in case of non-normal distribution they were given as median 25-75 percentiles (IQR). Categorical variables were depicted as percentages and numbers. We compared subjects according to median value of SYNTAX Score. Primary outcome was SYNTAX Score which was calculated by angiographic images.

The association between SYNTAX Score and the presence of age, gender, hypertension, DM, hyperlipidaemia (HL), smoking, systolic blood pressure, diastolic blood pressure, heart rate, haemoglobin, low-density lipoprotein (LDL), CR, BMI, BFP, RFM variables was evaluated using univariable linear regression models. Those variables with very high (95\%) and very low $(<5 \%)$ frequencies were not included in the model. Independent predictors to be included in the model are clinically and biologically plausible and that their association with SYNTAX has been demonstrated in previous studies and the stepwise backward univariate analysis of the present study. ${ }^{19}$

The association between SYNTAX Score and the presence of age, hypertension, DM, smoking, haemoglobin, LDL, CR, RFM variables was evaluated using linear regression models (model-1 RFM). Those variables with very high and very low frequencies were not included in the model.

In model 2, we use BFP instead of RFM but other predictor variables were the same. In model 3, we used BMI instead of RFM but other predictor variables were the same. Stepwise backward model was performed for RFM model. After switching from full model to reduced model, only DM and RFM were present and for predicting SYNTAX Score, and full model likelihood ratio was compared with reduced model likelihood ratio.

The relative importance of each predictor in the models was estimated with partial $\chi^{2}$ value for each predictor divided by the model's total $\chi^{2}$, which estimates the independent contribution of the predictor to the variance of the outcome. The performance of the model was measured by R-squared $\left(\mathrm{R}^{2}\right)$, Akaike's information criterion (AIC), Bayesian information criterion (BIC) and root mean square error (RMSE). The comparison between models was made with the assessment of fit (likelihood ratio $\chi^{2}$ ), quality (AIC and BIC) and predictive accuracy $\mathrm{R}^{2}$. Independent contribution of the predictors to the variance of outcome was estimated. In this regard, the relative importance of each predictor in the model RFM, BMI and BFP was estimated with partial $\mathrm{x}^{2}$ value for each predictor. Model performance calibration was evaluated by plotting the observed outcome on the $y$-axis. Deviation from the $45^{\circ}$ line indicates bias for the predicted outcome. In all the statistical analyses, a $p<0.05$ was considered as statistically significant.

\section{RESULTS}

A total of 325 patients were included in the study. Baseline demographic informations, blood sample parameters, anthropometric
Table 1 Baseline characteristics of study population according to SYNergy between percutaneous coronary intervention with TAXus and cardiac surgery trial (SYNTAX) Scores

\begin{tabular}{|c|c|c|c|}
\hline Variables & $\begin{array}{l}\text { SYNTAX group } 1 \\
(\mathrm{n}: 169)\end{array}$ & $\begin{array}{l}\text { SYNTAX group } 2 \\
(\mathrm{n}: 156)\end{array}$ & $P$ value \\
\hline Age (years) & $60.2 \pm 11.7$ & $63.5 \pm 10.3$ & 0.006 \\
\hline Gender (n, \%) (male) & $97, \% 57.4$ & $76, \% 48.7$ & 0.12 \\
\hline $\operatorname{DM}(n, \%)$ & $56, \% 33.1$ & $86, \% 55.1$ & 0.001 \\
\hline HT $(n, \%)$ & $77, \% 45.6$ & $81, \% 51.9$ & 0.025 \\
\hline $\mathrm{HL}(\mathrm{n}, \%)$ & $43, \% 25.4$ & $60, \% 38.5$ & 0.001 \\
\hline Systolic blood pressure (mm Hg) & $134.1 \pm 23.2$ & $134.3 \pm 19.3$ & 0.96 \\
\hline $\begin{array}{l}\text { Diastolic blood pressure (mm } \\
\mathrm{Hg})\end{array}$ & $75.2 \pm 13.1$ & $74.2 \pm 12.2$ & 0.46 \\
\hline Heart rate (beat/min) & $73.5 \pm 16.7$ & $77.3 \pm 16.3$ & 0.04 \\
\hline Smoking (n, \%) & $74, \% 43.8$ & $84, \% 53.8$ & 0.07 \\
\hline Glucose (mg/dL) & $121.5 \pm 53.9$ & $134.9 \pm 54.6$ & 0.03 \\
\hline $\mathrm{CR}(\mathrm{mg} / \mathrm{dL})$ & $0.88 \pm 0.27$ & $0.89 \pm 0.34$ & 0.92 \\
\hline Ure (mg/dL) & $36.6 \pm 14.6$ & $39.9 \pm 19.6$ & 0.08 \\
\hline Total cholestrol (mg/dL) & $189.9 \pm 43.8$ & $194.9 \pm 52.7$ & 0.34 \\
\hline Trigliseride (mg/dL) & $153.3 \pm 87.06$ & $132.5 \pm 87$ & 0.55 \\
\hline $\mathrm{LDL}(\mathrm{mg} / \mathrm{dL})$ & $119.1 \pm 38.3$ & $122.2 \pm 42.8$ & 0.49 \\
\hline White blood cell & $8.41 \pm 3.13$ & $8.57 \pm 2.36$ & 0.63 \\
\hline Haemoglobin (g/dL) & $13.3 \pm 1.76$ & $12.5 \pm 1.1$ & 0.001 \\
\hline Platelet & $246.8 \pm 94.6$ & $260.3 \pm 92.6$ & 0.24 \\
\hline $\mathrm{EF} \%$ & $56.0 \pm 6.7$ & $55.3 \pm 6.8$ & 0.35 \\
\hline Height $(\mathrm{cm})$ & $168.3 \pm 7.9$ & $164.6 \pm 8.8$ & 0.001 \\
\hline Weight (kg) & $81.7 \pm 14.3$ & $81.5 \pm 13.7$ & 0.91 \\
\hline Waist (cm) & $97.0 \pm 12.0$ & $98.8 \pm 13.2$ & 0.19 \\
\hline Hip (cm) & $96.2 \pm 12.7$ & $101.7 \pm 12.7$ & 0.001 \\
\hline BMI & $29.1 \pm 5.4$ & $30.1 \pm 5.1$ & 0.09 \\
\hline BFP & $36.9 \pm 6.4$ & $38.5 \pm 6.9$ & 0.02 \\
\hline BFM & $30.9 \pm 9.2$ & $32.0 \pm 9.3$ & 0.32 \\
\hline RFM & $31.2 \pm 6.8$ & $36.2 \pm 7.9$ & 0.001 \\
\hline
\end{tabular}

BFM, body fat mass; BFP, body fat percentage; BMI, body mass index; $C R$, creatinine; $\mathrm{DM}$, diabetes mellitus; $\mathrm{EF}$, ejection fraction; $\mathrm{HL}$, hyperlipidaemia; $\mathrm{HT}$, hypertension; LDL, low-density lipoprotein; RFM, relative fat mass.

measurements, BMI, BFP and RFM have been in two groups devided according to median SYNTAX (median SYNTAX : 11) Score of study sample in table 1.

Univariate linear regression outcome variable SYNTAX was used to determine whether there was any relationship between age, gender, DM, HT, HL, LDL, smoking, CR, haemoglobin, BFP, BMI and RFM. Based on our univariate analysis and the results reported in previous studies, 12 physiological and clinical plausible variables were selected as confounders including age, gender, DM, HT, HL, LDL, CR, haemoglobin, smoking, BFP, BMI and RFM (table 2).

They were included in the multivariable linear regression model 1 (model RFM). The analysis showed that in model 1 , RFM ( $\beta$ coefficient: 2.31 (0.90 to 3.71), $p=0.001)$ ), DM ( $\beta$ coefficient: 3.72 (1.67 to 3.76), $\mathrm{p}=0.004)$ ), HB ( $\beta$ coefficient: $-2.12(-3.70$ to -0.53$), p=0.009)$, age $(\beta$ coefficient: 1.83 (0.29 to 3.37), $\mathrm{p}=0.02))$ and smoking ( $\beta$ coefficient: 2.95 (0.92 to 4.98$), p=0.04)$ ) were statistically significant with increasing SYNTAX Scores while the other variables were not statistically significant (table 3, model 1).

Multivariable linear regression model 2 (model BFP) outcome variable was used to determine whether there was any relationship, independent from other confounders, between SYNTAX Score. Variables were chosen as confounders, like model 1, and 
Table 2 Univariate linear regression analysis between SYNergy between percutaneous coronary intervention with TAXus and cardiac surgery trial (SYNTAX) and variables

\begin{tabular}{lccc}
\hline & B coefficient & \multicolumn{1}{c}{$95 \%$ Cl } & P value \\
\hline Age (54-70) & 2.66 & 1.16 to 4.17 & 0.01 \\
\hline Gender (reference male) & 1.56 & -0.56 to 3.69 & 0.15 \\
\hline HB (11.5-14.4) & -2.92 & -4.46 , to 1.37 & 0.002 \\
\hline CR (0.69-1.0) & -0.12 & -1.20 to 0.95 & 0.82 \\
\hline HT & 2.66 & 0.55 to 4.78 & 0.01 \\
\hline DM & 4.88 & 2.80 to 6.96 & $<0.001$ \\
HL & 1.98 & -0.29 to 4.26 & 0.09 \\
\hline Smoking & 2.29 & 0.17 to 4.41 & 0.03 \\
LDL (93-147) & -0.35 & -1.77 to 1.08 & 0.84 \\
\hline RFM (28.9-39.5) & 4.81 & 3.45 to 6.17 & $<0.001$ \\
\hline BFP (33.7-42.2) & 1.96 & 0.62 to 3.29 & 0.004 \\
\hline BMI (26.0-32.8) & 1.22 & -0.15 to 2.61 & 0.08 \\
\hline
\end{tabular}

$\mathrm{BFP}$, body fat percentage; $\mathrm{BMI}$, body mass index; $\mathrm{CR}$, creatinine; $\mathrm{DM}$, diabetes mellitus; $H B$, haemoglobin; $H L$, hyperlipidaemia; $H T$, hypertension; LDL, low-density lipoprotein; RFM, relative fat mass.

they were included in the binary logistic regression model. The analysis showed that BFP ( $\beta$ coefficient: $1.21(-0.07$ to 2.49 ], $\mathrm{p}=0.06)$ ) was nor statistically significant but DM ( $\beta$ coefficient: 4.01 (1.96 to 6.07$), \mathrm{p}<0.001)$ ), smoking ( $\beta$ coefficient: 2.81 (0.74 to 4.89$), \mathrm{p}=0.008)), \mathrm{HB}(\beta$ coefficient: $-2.43[-4.03$ to -0.84$), p=0.003)$ ), age ( $\beta$ coefficient: 2.14 (0.60 to 3.67), $\mathrm{p}=0.006)$ ) were statistically significant with increasing SYNTAX Scores while the other variables were not (table 3, model 2).

Multivariable linear regression model 3 (model BMI) outcome variable was used to determine whether there was any relationship, independent from other confounders, between SYNTAX Score. Variables were chosen as confounders, like model 1, and they were included in the binary logistic regression model. The analysis showed that BMI ( $\beta$ coefficient: 0.90 ( -0.92 to 2.73 ), $\mathrm{p}=0.33)$ ) was nor statistically significant but DM ( $\beta$ coefficient: 4.13 (2.07 to 6.19), $\mathrm{p}<0.001)$ ), smoking ( $\beta$ coefficient: 2.96 (0.88 to 5.05), $\mathrm{p}=0.005), \mathrm{HB}$ ( $\beta$ coefficient: $-2.45(-4.05$ to -0.86$), p=0.002)$, age ( $\beta$ coefficient: 2.25 (0.71 to 3.79$)$, $\mathrm{p}=0.004)$ ) were statistically significant with increasing SYNTAX Scores while the other variables were not (table 3, model 3).

The performance of all models has been demonstrated in table 4 .
Table 4 Performance measurements of models

\begin{tabular}{llllll}
\hline & Adjusted $\mathrm{R}^{2}$ & Likelihood ratio & AIC & BIC & RMSE \\
\hline BFP model & 0.137 & 56.9 & 2386 & 2418 & 8.92 \\
BMI model & 0.130 & 54.4 & 2380 & 2420 & 8.95 \\
RFM model & 0.155 & 64.1 & 2370 & 2410 & 8.82 \\
\hline
\end{tabular}

AIC, Akaike's information criterion; BFP, body fat percentage; BIC, Bayesian information criterion; BMI, body mass index; RFM, relative fat mass; RMSE, root mean square error.

AIC, BIC and RMSE values of model 1 were lower than models 2 and 3 . Also, adjusted $\mathrm{R}^{2}$ value of model 1 was higher than models 2 and 3 (0.155, 0.137 and 0.130 , respectively); and $\chi^{2}$ values of RFM were higher than BFP and BMI $(10.5,3.4$ and 1.0 , respectively) (figure 2). Partial effect plot of the RFM in model $1, \mathrm{BFP}$ in model 2 and BMI in model 3 has been presented in figure 3 .

Stepwise backward model was performed for RFM model. After switching from full model to reduced model, only DM and RFM were present. For predicting SYNTAX Score, full model likelihood ratio was compared with reduced model likelihood ratio, and there was not a statistically significant difference between them $(p=0.29)$.

We performed corrected calibration plot for our models, fairly agreement with the apparent calibration was seen in model RFM better than other models (figure 4).

\section{DISCUSSION}

Our study showed that RFM, unlike BFP and BMI, could significantly predict SYNTAX Scores. RFM and CAD severity associations were out of obesity paradox terminology.

The WHO defined obesity as the increased percentage of body fat, and declared it a global epidemic due to its alarming prevalence rate. ${ }^{22}$ Increased adipose tissue can lead to CAD through direct effects on the cardiovascular system or through different disease mechanisms. It shows its effects through mechanisms such as increase in free fatty acid circulation, low-grade inflammation, endothelial dysfunction and metabolic disorder. While many comorbid factors associated with obesity and obesityrelated factors are known to pose a risk for CAD, the concept of the obesity paradox is confusing.

BMI is a parameter determined by the ratio of weight to height squared, which was defined by a Belgian astronomer and statistician Mr. Quetelet about 200 years ago; this arithmetic

Table 3 Multivariable linear regression model 1-model 2-model 3 comparison.

\begin{tabular}{|c|c|c|c|c|c|c|c|c|c|}
\hline & \multicolumn{3}{|c|}{ Model 1 (RFM) } & \multicolumn{3}{|l|}{ Model 2 (BFP) } & \multicolumn{3}{|l|}{ Model 3 (BMI) } \\
\hline & $\beta$ coefficient & $95 \% \mathrm{Cl}$ & $P$ value & $\beta$ coefficient & $95 \% \mathrm{Cl}$ & $P$ value & $\beta$ coefficient & $95 \% \mathrm{Cl}$ & $P$ value \\
\hline Age (54-70) & 1.83 & 0.29 to 3.37 & 0.02 & 2.14 & 0.60 to 3.67 & 0.006 & 2.25 & 0.71 to 3.79 & 0.004 \\
\hline Gender (male) & -1.69 & -4.15 to 0.76 & 0.17 & 0.53 & -1.53 to 2.61 & 0.61 & 0.41 & -1.69 to 2.54 & 0.7 \\
\hline HB (11.5-14.4) & -2.12 & -3.70 to -0.53 & 0.009 & -2.43 & -4.03 to 0.84 & 0.003 & -2.45 & -4.05 to 0.86 & 0.002 \\
\hline CR (0.69-1.0) & -0.72 & -1.76 to 0.32 & 0.17 & -0.78 & -1.83 to 0.26 & 0.38 & -0.77 & -1.83 to 0.27 & 0.14 \\
\hline HT & 0.92 & -1.09 to 2.95 & 0.36 & 1.0 & -1.04 to 3.05 & 0.33 & 1.04 & -1.01 to 3.09 & 0.31 \\
\hline DM & 3.72 & 1.67 to 5.76 & 0.004 & 4.01 & 1.96 to 6.07 & $<0.001$ & 4.13 & 2.07 to 6.19 & $<0.001$ \\
\hline Smoking & 2.95 & 0.92 to 4.98 & 0.04 & 2.81 & 0.74 to 4.89 & 0.008 & 2.96 & 0.88 to 5.05 & 0.005 \\
\hline $\mathrm{HL}$ & 0.65 & -1.51 to 2.81 & 0.55 & 0.79 & -1.38 to 2.97 & 0.47 & 0.79 & -1.39 to 2.98 & 0.47 \\
\hline RFM (28.9-39.5) & 2.31 & 0.90 to 3.71 & 0.001 & & & & & & \\
\hline BFP (33.7-42.2) & & & & 1.21 & -0.07 to 2.49 & 0.06 & & & \\
\hline BMI (26-32.7) & & & & & & & 0.68 & -0.65 to 1.99 & 0.32 \\
\hline
\end{tabular}

BFP, body fat percentage; $B M I$, body mass index; $C R$, creatinine; $D M$, diabetes mellitus; $H B$, haemoglobin; $H L$, hyperlipidaemia; $H T$, hypertension; RFM, relative fat mass. 

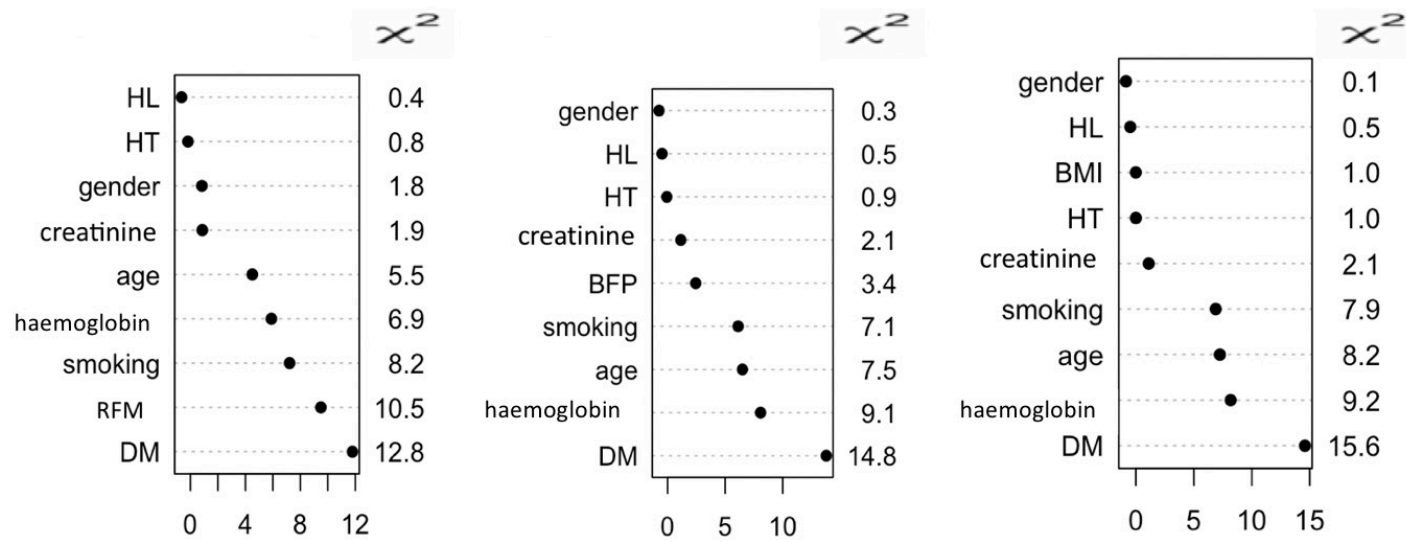

Figure 2 Partial effect plot for SYNTAX Score in overall population. BFP, body fat percentage; BMI, body mass index; DM, diabetes mellitus; HL, hyperlipidaemia; HT, hypertension; RFM, relative fat mass; SYNTAX, SYNergy between percutaneous coronary intervention with TAXus and cardiac surgery trial.

approach has been used extensively to define the limits of obesity and to categorise people, but the fact that this formula is not a measure of adiposity and is merely a mathematical equation is a major error and can cause misclassification. ${ }^{23}$ Since BMI cannot directly measure BFP and distinguish between total body fat and total body weight, BMI is an imperfect measure of body fatness. When we compare two people with the same BMI, one may have a high body fat mass and less muscle mass and the other can have a low fat mass with high muscle mass. According to the BMI, we cannot compare the body fat and we might misclassify people as obese or non-obese.

The relationship between BMI and CAD severity is controversial in some studies, despite higher prevalence of recognised risk factors and increased incidence of adverse cardiovascular events. According to the BMI formula, the correlation between obesity and survival was defined as a U-shaped curve. ${ }^{24}$ It has been shown in many studies that there is a paradoxical correlation between BMI and CAD severity. This has led to the definition of obesity paradox in cardiovascular diseases. In a meta-analysis of 40 cohort studies with the participation of more than 250000 patients, it was reported that overweight/ obese people with CAD had a lower risk of total and cardiovascular mortality compared with underweight and normalweight patients. ${ }^{25}$ An additional large meta-analysis of 89 studies including more than 1.3 million patients with CAD also confirmed an obesity paradox with CAD. ${ }^{26}$ Rubinshtein et al and Niraj et al showed that obesity is associated with less severe CAD in patients undergoing CAG. ${ }^{910}$ Studies have shown that the poor prognosis-related comorbidities such as diabetes and hypertension are more common in patients with high BMI, but the condition defined as the obesity paradox is related to BMI independent of these comorbidities. Despite these studies pointing to the obesity paradox, some studies showed the positive relationship between obesity and cardiovascular diseases; these confusing results led to questioning the diagnosis of obesity using BMI formula. ${ }^{27}$
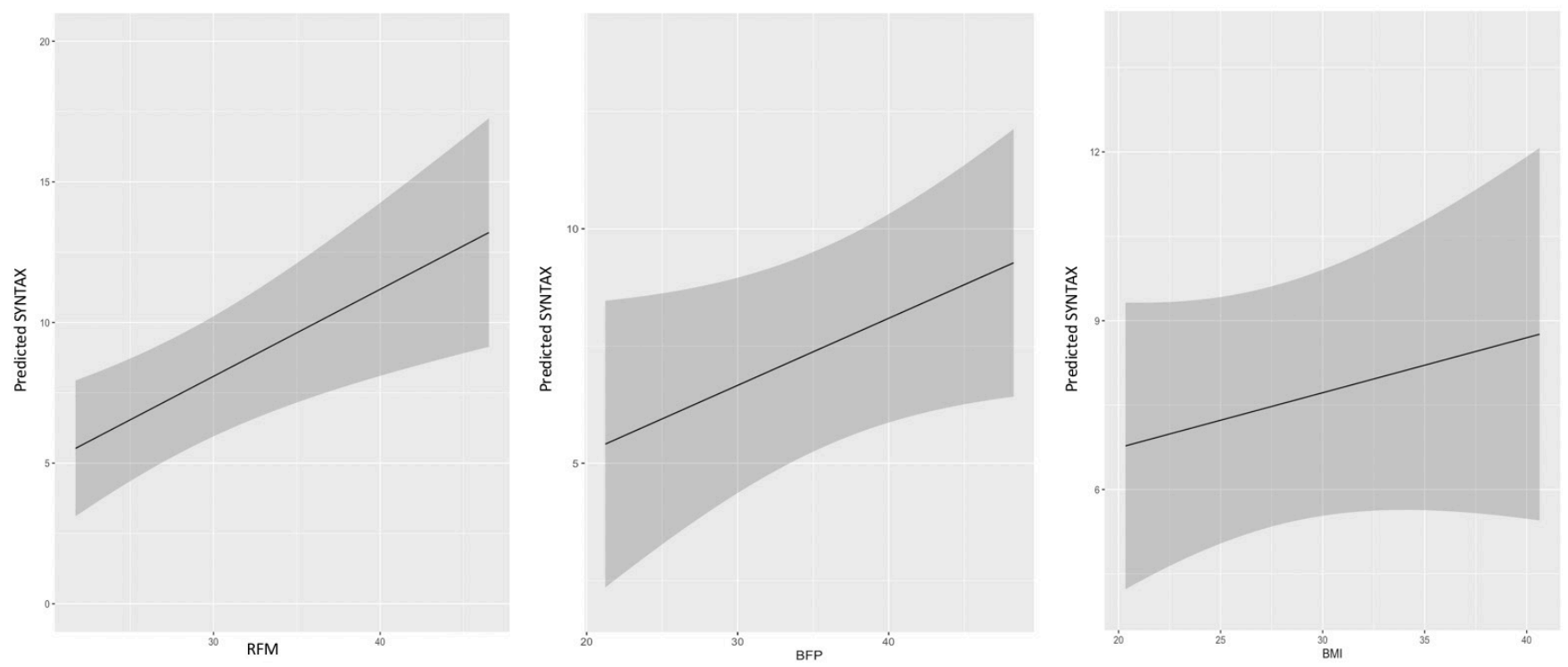

Figure 3 The relative importance of each predictor in the models. BFP, body fat percentage; BMI, body mass index; RFM, relative fat mass; SYNTAX, SYNergy between percutaneous coronary intervention with TAXus and cardiac surgery trial. 
RFM

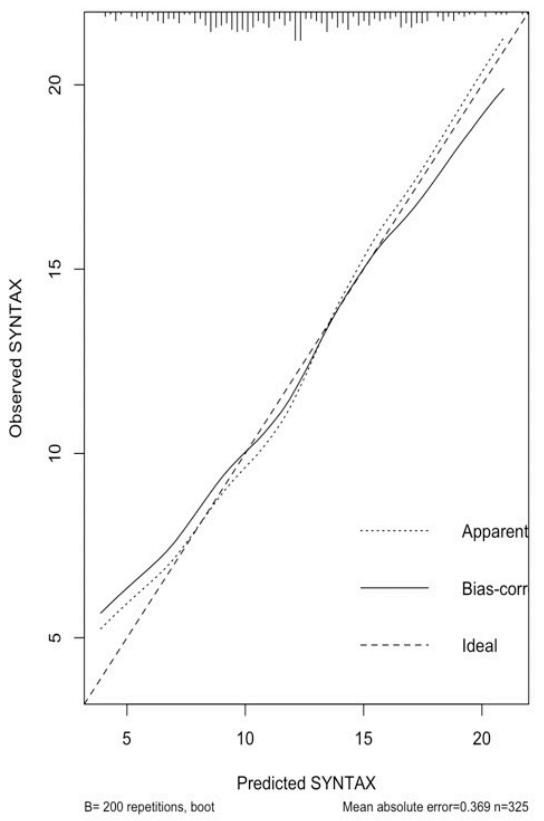

BFP

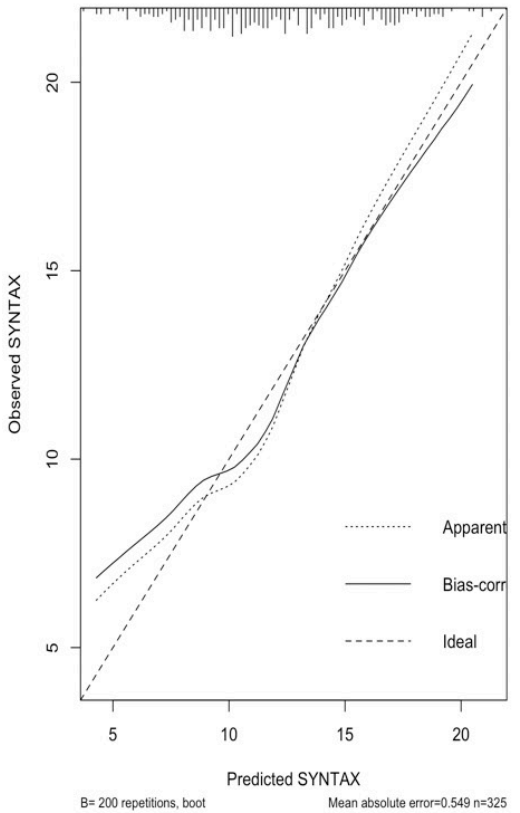

BMI

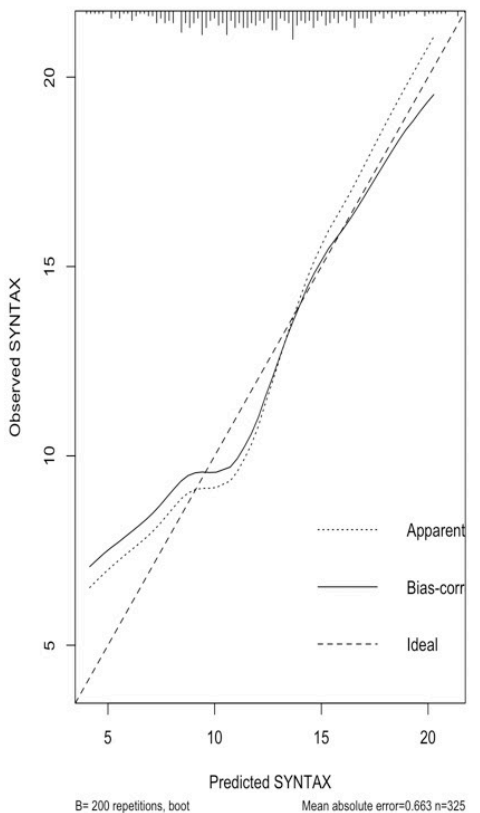

Figure 4 Corrected calibration plot. BFP, body fat percentage; BMI, body mass index; RFM, relative fat mass; SYNTAX, SYNergy between percutaneous coronary intervention with TAXus and cardiac surgery trial.

In addition to the BMI measurement, the BFP formula is used to determine the body fat ratio. In some studies, patients with CAD and increased BFP have a higher risk for major adverse cardiovascular events. BFP values have been shown to be more valuable in predicting cardiovascular outcomes than $\mathrm{BMI} .{ }^{28} \mathrm{On}$ the other hand, in some studies obesity paradox exists and BFP gives similar results as BMI values. ${ }^{29}$ The results of a study which evaluated coronary artery calcification (CAC) in computer tomography indicated that higher BFP was associated with higher incidence of CAC only in men. ${ }^{30}$ Although there is no study comparing the prevalence of CAD with BFP using CAG; in our study, BFP was little assosciated with the prevalence of CAD but its predictive power remained behind RFM measurements.

In studies which show obesity paradox, the better prognosis of patients with obesity can be explained by examination and treatment in the early stages of the disease course. Patients with a high BMI also resort to standard medical treatments, diagnostic CAG, and revascularisation procedures more frequently and at an early age. Since these patients are younger, the prevalence of other cardiovascular risk factors is lower; patients with obesity have lower CAD burden and lower prevalence of high-risk coronary anatomy (left main coronary or three vessel disease) than nonobese patients. ${ }^{9}{ }^{10}$ In addition, the absence of high-risk patients in some studies makes the association between BMI and CAD difficult. In these studies, instead of presenting these factors as an excuse, it is more important to question the BMI formula used in the diagnosis of obesity.

As a result of confusing studies, a new proposal was made to estimate the WBF and to define the obesity known as RFM Index. RFM is calculated using height, WC and gender, regardless of weight. In some studies, it has been shown that the percentage of WBF measured by dual energy X-ray absorptiometry (DXA) between women and men is better predicted by RFM Index than BMI. Also, RFM is known to be superior to $\mathrm{BMI}$ in determining risk for DM. ${ }^{18}$ RFM provides better predictability for dyslipidaemias and metabolic syndrome than BMI. ${ }^{31}$ RFM is superior to BMI in predicting independent risk factors for CAD, like DM, dyslipidaemias and metabolic syndrome. This may suggest that RFM is a more reliable parameter than BMI about total cardiovascular disease burden and frequency estimation.

While obesity is an indicator of body fat, it is questionable how much the BMI formula used in its definition can evaluate it. Therefore, it is thought that body fat components can be determined more accurately by using the newly defined RFM formula. In our study, we found that the results that can normally be defined as the obesity paradox were rationally concluded using the RFM formula.

When obesity classification was made according to RFM and BMI values in our study, we observed that more patients fell into the obesity classification by RFM. The severity of CAD calculated by SYNTAX Score in patients was significantly higher in RFM than BFP and BMI classification groups. Although other values were constant, $\mathrm{R}^{2}$ value was higher in model RFM, which shows that RFM model is more explanatory for describing SYNTAX Score relationship (figure 2; table 4). Also, in RFM model, AIC and BIC values were lower than other models, which shows that accuracy is higher in RFM model.

In our study, we attempted to compare the efficacy of RFM, BFP, BMI and some anthropological parameters in determining the severity of CAD defined by CAG. The usability of the BMI formula that has been used for years in the definition of obesity should be questioned. It has been shown in our study that the parameters in the BMI formula are not sufficient to show the body fat ratio, as in similar publications in the literature. Determining the increase in body fat ratio, which is an important comorbidity in CAD, with new formulas such as RFM may bring the obesity paradox phenomenon back to the agenda. Our study has been a study questioning the obesity paradox using RFM in cardiovascular diseases. We observed that RFM values were associated better with the severity of CAD and were more powerful in showing the relationship between obesity and CAD. Obesity 
paradox phenomenon can be solved by using RFM in studies related to obesity and obesity-related cardiovascular diseases.

\section{CONCLUSION}

The results of our study showed that RFM Index is a more reliable and compatible marker of obesity for predicting the severity of CAD compared with BFP and BMI.

\section{LIMITATIONS}

In our study, if we could use DXA to show body fat rates, we would have supported our results more strongly. In addition, acute coronary syndromes were not included in the study, and the results of our study should be supported by studies with larger sample sizes.

\section{Main messages}

- Confusion caused by irrelevance between body mass index (BMI) and obesity led the researchers to seek for new equations. As a result of the studies, a new proposal was made to estimate the whole body fat and to define obesity, which is known as relative fat mass (RFM).

- Since BMI cannot directly measure body fat percentage and distinguish between total body fat and total body weight, $\mathrm{BMI}$ is an imperfect measure of body fatness. RFM Index is calculated using height, waist circumference and gender regardless of weight. In some studies, it has been shown that the percentage of whole body fat is better predicted by RFM than BMI.

- We observed that RFM values were associated more consistent with the severity of coronary artery disease (CAD) and were more powerful in showing the relationship between obesity and CAD. Obesity paradox phenomenon could be solved by using RFM in studies related to obesity and obesityrelated cardiovascular diseases.

\section{Current research questions}

- RFM is a better formulation than BMI at predicting total fat mass. More reliable results can be obtained with the use of RFM instead of BMI, in new studies on many subjects known as obesity parodoxia.

\section{What is already known on the subject}

- It is known that being overweight and obese are associated with an increased risk of cardiovascular disease, that can cause mortality increase in the general population.

- Although different approaches have attempted to define obesity to date, there is no consensus about its exact definition yet, BMI has been used frequently to determine definitions for obesity limits.

- It has been shown in many studies that there is a paradoxical correlation between BMI and cardiovascular diseases. This has led to the definition of obesity paradox in cardiovascular diseases.

Contributors Concept: SCE, NO and TK. Design: SCE, BA and SK. Data collection or processing: CD, SK, YY, MSA, ZB and SCE. Analysis or interpretation: SCE, AK and NO. Literature search: SCE, SK, AK and NO. Writing: SCE and all authors controls.

Funding The authors have not declared a specific grant for this research from any funding agency in the public, commercial or not-for-profit sectors.
Competing interests None declared.

Patient consent for publication Not required.

Ethics approval The research protocol of this study was approved by the Research Review Committee of the Department of Cardiology and the Ethical Committee prior to starting the research.

Provenance and peer review Not commissioned; externally peer reviewed.

Data availability statement Data are available upon reasonable request. Data are available upon reasonable request.

\section{ORCID iD}

Süleyman Çağan Efe http://orcid.org/0000-0002-6067-6841

\section{REFERENCES}

1 Haslam DW, James WPT. Obesity. Lancet 2005;366:1197-209.

2 Berrington de Gonzalez A, Hartge P, Cerhan JR, et al. Body-Mass index and mortality among 1.46 million white adults. N Engl J Med 2010;363:2211-9.

3 Dudina A, Cooney MT, Bacquer DD, et al. Relationships between body mass index, cardiovascular mortality, and risk factors: a report from the score Investigators. Eur $\int$ Cardiovasc Prev Rehabil 2011;18:731-42.

4 Lavie CJ, Kachur S, De Schutter A. Is there an obesity paradox in coronary heart disease in Asia? Coron Artery Dis 2017;28:273-4.

5 Vecchié A, Dallegri F, Carbone F, et al. Obesity phenotypes and their paradoxical association with cardiovascular diseases. Eur J Intern Med 2018;48:6-17.

6 Singh RB, Rastogi SS, Niaz MA, et al. Association of central obesity and insulin resistance with high prevalence of diabetes and cardiovascular disease in an elderly population with low fat intake and lower than normal prevalence of obesity: the Indian paradox. Coron Artery Dis 1998;9:559-66.

7 Hastie CE, Padmanabhan S, Slack R, et al. Obesity paradox in a cohort of 4880 consecutive patients undergoing percutaneous coronary intervention. Eur Heart J 2010;31:222-6.

8 Diercks DB, Roe MT, Mulgund J, et al. The obesity paradox in non-ST-segment elevation acute coronary syndromes: results from the can rapid risk stratification of unstable angina patients suppress adverse outcomes with early implementation of the American College of Cardiology/American heart association guidelines quality improvement initiative. Am Heart J 2006;152:140-8.

9 Das SR, Alexander KP, Chen AY, et al. Impact of body weight and extreme obesity on the presentation, treatment, and in-hospital outcomes of 50,149 patients with ST-segment elevation myocardial infarction results from the NCDR (national cardiovascular data registry). J Am Coll Cardiol 2011;58:2642-50.

10 Park HW, Kim K-H, Song IG, et al. Body mass index, carotid plaque, and clinical outcomes in patients with coronary artery disease. Coron Artery Dis 2017;28:278-86

11 Aune D, Sen A, Prasad M, et al. Bmi and all cause mortality: systematic review and non-linear dose-response meta-analysis of 230 cohort studies with 3.74 million deaths among 30.3 million participants. BMJ 2016;4:i2156.

12 Shehab A, Al-Dabbagh B, AlHabib K, et al. The obesity paradox in patients with acute coronary syndrome: results from the Gulf RACE-2 study. Angiology 2014;65:585-9.

13 Rubinshtein $\mathrm{R}$, Halon DA, Jaffe $\mathrm{R}$, et al. Relation between obesity and severity of coronary artery disease in patients undergoing coronary angiography. Am J Cardiol 2006;97:1277-80.

14 Labounty TM, Gomez MJ, Achenbach S, et al. Body mass index and the prevalence, severity, and risk of coronary artery disease: an international multicentre study of 13,874 patients. Eur Heart J Cardiovasc Imaging 2013;14:456-63.

15 Schulte H, Cullen P, Assmann G, Obesity AG. Obesity, mortality and cardiovascular disease in the Münster heart study (PROCAM). Atherosclerosis 1999;144:199-209.

16 Ryan DH, Kahan S. Guideline recommendations for obesity management. Med Clin North Am 2018;102:49-63.

17 Garvey WT, Mechanick Jl, Brett EM, et al. American association of clinical endocrinologists and American College of endocrinology comprehensive clinical practice guidelines for medical care of patients with obesity. Endocr Pract 2016;22 Suppl 3:1-203

18 Woolcott 00, Bergman RN. Relative fat mass (RFM) as a new estimator of wholebody fat percentage - a cross-sectional study in American adult individuals. Sci Rep 2018;8:10980.

19 Sianos G, Morel M-A, Kappetein AP, et al. The SYNTAX score: an angiographic tool grading the complexity of coronary artery disease. Eurolntervention 2005;1:219-27.

20 Gensini GG. A more meaningful scoring system for determining the severity of coronary heart disease. Am J Cardiol 1983;51:606.

21 Kachur S, Lavie CJ, de Schutter A, et al. Obesity and cardiovascular diseases. Minerva Med 2017;108:212-28.

22 WHO. Obesity: preventing and managing the global epidemic, 2000.

23 Allison DB, Zhu SK, Plankey M, et al. Differential associations of body mass index and adiposity with all-cause mortality among men in the first and second National health and nutrition examination surveys (NHANES I and NHANES II) follow-up studies. Int J Obes Relat Metab Disord 2002;26:410-6. 
24 Romero-Corral A, Montori VM, Somers VK, et al. Association of bodyweight with total mortality and with cardiovascular events in coronary artery disease: a systematic review of cohort studies. Lancet 2006;368:666-78.

25 Wang ZJ, Zhou YJ, Galper BZ, et al. Association of body mass index with mortality and cardiovascular events for patients with coronary artery disease: a systematic review and meta-analysis. Heart 2015;101:1631-8.

26 Widecka K, Safranow K, Lewandowski M, et al. Angiographic severity of coronary artery disease and cardiovascular risk in acute coronary syndrome in patients with metabolic syndrome. Kardiol Pol 2018;76:662-8.

27 Medina-Inojosa JR, Somers VK, Thomas RJ, et al. Association between adiposity and lean mass with long-term cardiovascular events in patients with coronary artery disease: no paradox. J Am Heart Assoc 2018;7:pii: e007505.
28 De Schutter A, Lavie CJ, Patel DA, et al. Relation of body fat categories by Gallagher classification and by continuous variables to mortality in patients with coronary heart disease. Am J Cardiol 2013;111:657-60.

29 Huang B-T, Peng Y, Liu W, et al. Lean mass index, body fat and survival in Chinese patients with coronary artery disease. QJM 2015;108:641-7.

30 Aljizeeri A, Coutinho T, Pen A, et al. Obesity and coronary artery calcification: can it explain the obesity-paradox? Int J Cardiovasc Imaging 2015;31:1063-70.

31 Kobo 0, Leiba R, Avizohar 0, et al. Relative fat mass is a better predictor of dyslipidemia and metabolic syndrome than body mass index. Cardiovasc Endocrinol Metab 2019;8:77-81. 